\title{
Cahit Arf: Exploring his scientific influence using social network analysis, author co-citation maps and single publication $h$ index ${ }^{1}$
}

\author{
Yaşar Tonta*, A. Esra Özkan Çelik ${ }^{1}$ \\ Department of Information Management, Faculty of Letters, Hacettepe University, 06800 Beytepe, Ankara, Turkey, \\ ${ }^{1}$ Registrar's Office, Hacettepe University, 06800 Beytepe, Ankara, Turkey
}

\begin{abstract}
Cahit Arf (1910-1997), a famous Turkish scientist whose picture is depicted in one of the Turkish banknotes, is a well-known figure in mathematics with his discoveries named after him (e.g., Arf invariant, Arf rings, the Hasse-Arf theorem). Although Arf may not be considered as a prolific scientist in terms of number of papers (he authored a total of 23 papers), his influence on mathematics and related disciplines was profound. As he was active before, during, and after World War II, Arf's contributions are not properly listed in citation indexes, and thus did not generate many citations even though several papers with "Arf" in their titles appeared in literature. This paper traces influence of Arf in scientific world using citation analysis techniques first. It reviews scientific impact of Arf by analyzing both; the papers authored by Arf and papers whose titles or keywords containing various combinations of "Arf invariant," "Arf rings," and so on. The paper then goes on to study Arf's contributions using social network analysis (SNA) and author co-citation analysis (ACA) techniques. CiteSpace and pennant diagrams are used to explore scientific impact of Arf by mapping his cited references derived from Thomson Reuters' Web of Science (WoS) database. The direct and indirect influences of Arf's highly cited paper on Arf invariant are assessed through ACA and single publication $h$ index, respectively. The paper ends with a discussion of whether data analysis techniques used in this study can be useful to study scientific impact of researchers retrospectively.
\end{abstract}

Keywords: Author co-citation analysis, Cahit Arf, CiteSpace, pennant diagrams, single publication $h$ index, social network analysis

\section{INTRODUCTION}

Cahit Arf is a world-renowned Turkish mathematician who has significant discoveries to his credit, which still are in use today such as "Arf invariant", "Arf rings", and "Arf closure". He was born in Selanik (Thessaloniki) on February 18, 1910. With the outbreak of Balkan Wars,

*Address for correspondence:

E-mail: tonta@hacettepe.edu.tr

\begin{tabular}{|l|l|}
\hline \multicolumn{2}{|c|}{ Access this article online } \\
\hline Quick Response Code: & \\
\cline { 1 - 1 } & Website: \\
\cline { 2 - 3 } & www.jscires.org \\
& \\
\hline
\end{tabular}

his family migrated to Istanbul in 1912, then to Ankara, and finally settled in Izmir. As a brilliant student, Arf successfully completed the École Normale Supérieure in Paris in 2 years and then worked as a teacher at Galatasaray High School for a year with great willingness. In 1933, he joined the Mathematics Department of Istanbul University as an assistant professor and finally began to work as a mathematician for academic purposes. Starting from 1933, Istanbul University became home to many German emigrated scientists who fled the Nazi regime in Germany. Among them were distinguished mathematicians such as Richard von Mises, William Prager, and Hilda Geiringer. ${ }^{[1,2]}$ Reisman ${ }^{[3]}$ provides more information on Turkey's

1. This is a slightly revised version of the paper presented at COLLNET 2011: $7^{\text {th }}$ International Conference on Webometrics, Informetrics and Scientometrics (WIS), September 20-23, Istanbul, Turkey. It appeared in the conference proceedings CD-ROM (pp. 499-521). 
modernization of its higher education system in 1933 and the involvement of some 190 German scientists in this process.

In 1937, Arf went to Göttingen to have his $\mathrm{PhD}$ degree under the supervision of Professor Helmut Hasse. He completed his doctoral studies in 1.5 years (1938); the Hasse-Arf theorem was an outcome of his doctoral thesis. After completing his $\mathrm{PhD}$, he stayed in Göttingen one more year at the request of Professor Hasse ${ }^{[4]}$ and began to study on quadratic forms over a field of characteristic two to improve the theory established by Ernst Witt (1937). ${ }^{[5]}$ In 1941, Arf published the results of his study and completed the theoretical gap by introducing an important invariant of quadratic forms over a field of characteristic two. ${ }^{[6]}$ This invariant is known as the Arf invariant. It is the key to solution of several classical and fundamental problems about topology of manifolds. For Arf, it was his introduction to the international world of mathematics. ${ }^{[7,8]}$ In 1948, Arf published another significant contribution in the Proceedings of the London Mathematical Society. ${ }^{[0]}$ The most significant follow up to Arf's paper came from Lipman ${ }^{[10]}$ who was the first mathematician to mention "Arf rings" in the literature. ${ }^{[1]}$

Besides his many significant discoveries in mathematics, Professor Arf worked in Istanbul University until his involvement, on invitation, in the foundation of the Scientific and Technological Research Council of Turkey (TUBITAK). He served as the head of TUBITAK's Science Council between 1963 and 1967. In 1963, he joined the Mathematics Department of Robert College in Istanbul and worked at the Institute for Advanced Studies in Princeton, New Jersey, for 2 years. While at Princeton, he was invited to spend a year at the University of California at Berkeley as a visiting scholar. Then, he made his final return to Turkey, joined the Mathematics Department of the Middle East Technical University and continued his studies there until his retirement in 1980 . He received the prestigious Inonu Award in 1948, TUBITAK Science Award in 1974, and Commandeur des Palmes Académiques (France) in 1994. Arf was a member of the Mainz Academy and the Turkish Academy of Sciences. He served as the president of the Turkish Mathematical Society between 1985 and 1989. After his many contributions to the international mathematics society and the Turkish scientific environment, he passed away on December 26, 1997 in Bebek, Istanbul, at the age of 87. ${ }^{[1]}$ (See also O'Connor and Robertson. $\left.{ }^{[12]}\right)$ In 2009, the banknote of 10 Turkish Lira was issued with Professor Cahit Arf's portrait depicted on it [Figure 1]. The two libraries of TUBITAK

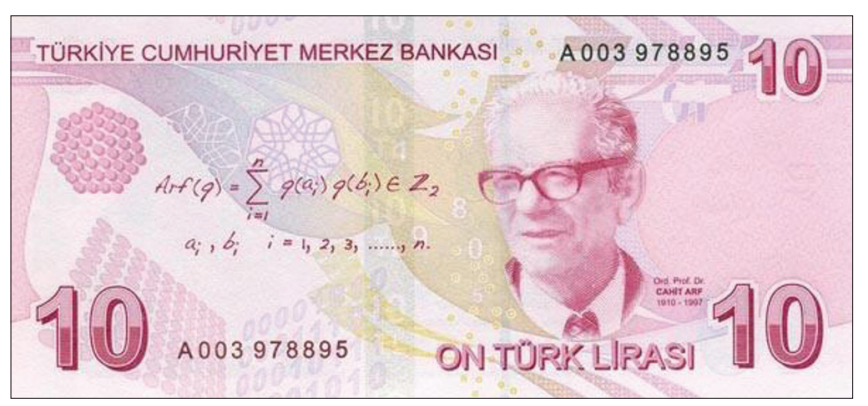

Figure 1: Professor Cahit Arf's portrait graces the reverse of the 10 Turkish lira banknote

bear Cahit Arf's name: "Cahit Arf Information Center" in Ankara (http://www.ulakbim.gov.tr/eng/cabim/) and "Cahit Arf Library" in Kocaeli nearby Istanbul (http:// www.mam.gov.tr/kutuphane/).

Within his 87 years life time, Arf was well known for the Arf invariant, Arf rings, Arf semi-groups, and the Hasse-Arf theorem, among others. This paper aims to study the influence of Cahit Arf's papers retrospectively by means of a combination of citation and ACA, social network analysis (SNA), and single publication $b$ index. Papers that referred to Arf's contributions in their titles and topics were identified, and an ACA was carried out. CiteSpace was used to find out Arf's place in mathematics and his impact on the basis of bibliometric analysis, ACA, and pennant diagrams. ${ }^{[13,14]}$ The indirect influence of Arf's seminal paper was calculated using Schubert's single publication $b$ index. ${ }^{[15]}$ We discuss the consequences of such an approach and conclude that ACA, pennant diagrams, and single publication $b$ index can shed further light on the influence of authors and help put their works in full perspective.

\section{LITERATURE REVIEW}

SNA is used to study and visualize structures of social networks. Based on graph theory, SNA has been widely used to reveal relationships among documents, journals, and authors. ${ }^{[16]}$ Scientific and intellectual ties and collaboration between researchers can be identified using bibliometric data (e.g., citations). The structure of scientific disciplines as social networks can be mapped by means of visualization software such as CiteSpace. ${ }^{[17]}$

Author co-citation analysis (ACA) was first introduced by White and Griffith. ${ }^{[18]}$ ACA assumes that the two researchers being cited together in scientific literature are likely to share the same research interests and work in the same field. Researchers working in the same 
domain get clustered through ACA, thereby facilitating discovery of social structure among researchers as well as among research domains. The outcome of ACA studies were used to map and visualize the structure of several scientific disciplines including information science and macroeconomics (e.g., see McCain ${ }^{[19,20]}$ and White and $\left.\mathrm{McCain}^{[21}\right)$.

White recently combined ACA with information retrieval (IR) and relevance theory (RT) to study the influence of a seed work, author, or paper. ${ }^{[13,14,22,23]} \mathrm{He}$ used the weights of term frequency $(t f)$ and inverse document frequency (idf) formula to draw pennant diagrams for works co-cited with a seed work, the authors co-cited with a seed author or articles and books co-cited with a seed article. Originally, $t f$ values are used in IR to determine relevance of a term within a given document (the more a term is used in a single document, the higher its relevance to a given query) while $i d f$ values are used to determine its relevance within the entire document collection (the more a term is used in different documents in a collection, the less discriminatory; and therefore, topically less relevant it becomes). Sparck Jones $^{[24]}$ created $i d f$ as a measure for weighing "statistical specificity" of terms. The $i d f$ measure pushes related term's weight down in rankings so that terms that occur relatively frequently in the document collection are considered less relevant to a query.

Bibliometric data and IR techniques are used in pennant diagrams "to mimic a relevance theoretic model of cognition on the user side". ${ }^{[13]}$ Pennant diagrams are scatterplots of $t f$ values representing "cognitive effects" of works/authors/articles in context of a seed work/author/ article, and $i d f$ values representing the "processing effort" of the user. Cognitive effects $(t f)$ and ease of processing (idf ) of works, authors, or articles determine their relevance to a seed work, seed author, or seed article. ${ }^{[13]}$ Whereas $t f^{*}$ idf formula multiplies the two to come up with a single score of relevance; pennant diagrams plot $t f$ values on the $x$ axis and $i d f$ values on the $y$ axis without multiplication. ${ }^{[13]}$ See White ${ }^{[13]}$ for a more comprehensive discussion of theoretical foundation of bibliometrics, IR, and RT along with their use in three different types of pennant diagrams for a seed work, a seed author, and a seed article.

White used pennant diagrams innovatively to study the influence of a work (Moby Dick), an author (Howard D. White), and a paper (Stephen Harter's "Psychological Relevance and Information Science") and interpreted his findings based on ACA, IR, and RT. ${ }^{[13]}$ Pennant diagrams proved to be useful in discovering new relationships between works, authors, and papers.

Metrics such as citation indicators and $b$ index originally proposed by Hirsch ${ }^{[25]}$ measure the direct influence of authors and papers as well as journals, institutions, and so on; but usually ignore their indirect influences. Schubert ${ }^{[15]}$ proposed the single publication $b$ index for highly cited papers, taking their indirect influence into account through citing papers. The higher the $b$ index of the set of papers citing a particular paper in question, the higher its single publication $h$ index score becomes even though the work in question is not cited directly. Recently, Thor and Bornmann $^{[2]}$ developed a web application ${ }^{2}$ to calculate single publication $b$ index of a paper using Schubert's definition and Google Scholar data.

It is fitting to review works on Cahit Arf in this study briefly. A detailed Turkish biography including his memoirs as well as his colleagues' views on his work and personality was written by Terzioğlu and Yllmaz and published by the Turkish Academy of Sciences in 2005. ${ }^{[1]}$ The book also includes some of his papers on mathematics as well as his writings on more general issues such as education. A scientific biography of Arf was written by Sertöz, ${ }^{[27]}$ who also maintains a website with pointers to papers (mostly in Turkish) about Arf as well as about other famous Turkish mathematicians. ${ }^{3}$ A special issue on Arf was published after his death by TUBITAK's science and technology magazine, Bilim ve Teknik. ${ }^{[28]}$ The special issue comprises several articles about Arf by some of his colleagues who met him or worked with him during his long career. To honor Arf's $80^{\text {th }}$ birthday, a monograph including all of his papers (along with four papers written by his colleagues about his works) was published by the Turkish Mathematical Society. ${ }^{[29]}$ The Mathematics Department of the Middle East Technical University organizes "Cahit Arf Lectures" annually.

As for books and papers based on Cahit Arf's works, they are too numerous to review in this paper. For instance, there are books that are entirely or in large part on Arf invariant and its generalizations (e.g., Snaith, ${ }^{\left[{ }^{30]}\right.}$ Klaus, ${ }^{[31]}$ Scorpan, ${ }^{[32]}$ Kirby, ${ }^{[33]}$ and Browder) ${ }^{[34]}$ Recently, Lorenz and Roquette $^{[35]}$ investigated the Arf invariant in its historical context based on letters exchanged between Cahit Arf and his advisor, Professor Helmut Hasse, after Arf got his $\mathrm{PhD}$ from Göttingen University. Interested readers can find further information on similar works by consulting the

2. Available freely at http://labs.dbs.uni-leipzig.de/gsh.

3. http://www.bilkent.edu.tr/ sertoz/turkler.htm.

4. http://www3.iam.metu.edu.tr/matematikvakfi/arf.html. 
basic reference sources or databases, some of which are readily available through the web.

The present study is an attempt to explore scientific legacy of a world-famous Turkish mathematician, Cahit Arf. Since the scientific impact of Arf's works cannot be measured readily by using citation indexes, we use both SNA and White's approach of ACA coupled with pennant diagrams. The indirect influence of Arf's highly cited paper, "Untersuchungen über quadratische Formen in Körpern der Charakteristik 2 (Teil 1)", ${ }^{[6]}$ is also assessed through its single publication $b$ index. Results of SNA, ACA, and single publication $b$ index are discussed along with the implications of using these methods and metrics to study scientific impact of authors directly, indirectly, and retrospectively.

\section{MATERIALS AND METHODS}

We think the scientific legacy of Cahit Arf is underrepresented in Thomson Reuters' (formerly ISI's) citation indexes (1898-2011). Hence, we decided to address several research questions to paint a better picture of his accomplishments as a mathematician. For example, who were the authors being co-cited most often with Arf? How high were their $b$ index scores? Can we trace the scientific influence of Arf through paper titles and topics that contain the terms "Arf invariant," "Arf rings", and so on? Which paper of Arf received the highest number of citations? Will the pennant diagram of Arf's most frequently cited paper provide further insight into his influence in mathematics as well as in other disciplines? What is the single publication $b$ index of his most significant work?

To address these research questions, we first searched for bibliographic records for Cahit Arf ("Arf C*”) in the Web of Science (WoS). Search results were in no way satisfactory (for the reasons explained before) to study the influence of Arf's contributions. We then searched for records having "Arf*" in their titles and/or topics and analyzed them using CiteSpace, which is a freely available application developed by $\mathrm{Chen}^{[17]}$ to analyze scientific literature and visualize trends and patterns in the data. ${ }^{5}$ We studied the distribution of citations on the co-citation network derived from CiteSpace by time-slicing them in 10-year intervals, to identify other influential mathematicians who were co-cited with Arf.

We searched for cited references of Arf from WoS and found that his paper on what is later called "Arf invariant"

5. http://cluster.cis.drexel.edu/ cchen/citespace/ was cited a total of 105 times. ${ }^{[6]}$ Then, using $\operatorname{Arf}^{\left[{ }^{[6]}\right.}$ as the seed work, we mapped ACA results onto a pennant diagram using White's approach. ${ }^{[13,14]}$ To create Arf's pennant diagram, we used the most highly cited $20 \%$ of references contained in 100 papers citing Arf. ${ }^{[6]}$ Using Arf's pennant diagram, we identified other influential authors in the author co-citation network whose work was most relevant to those of Arf, and more specifically, to Arf's seed work. ${ }^{[13]}$ Finally, we calculated the single publication $b$ index of Arf's highly cited work ${ }^{[6]}$ on the basis of Google Scholar data and tried to ascertain its indirect influence based on its citing papers. ${ }^{[15,26]}$ We used WoS to identify the top 50 mathematics papers that received highest number of citations and compared their single publication $h$ indexes with that of Arf. ${ }^{[6]}$

\section{FINDINGS AND DISCUSSION}

In this section, we first present the findings of citation analysis of Arf's papers using Thomson Reuters' citation indexes. Our analysis includes results of both cited reference search under "Arf $C^{*}$ " and of an advanced search for papers that included "Arf" in their titles and keywords. We then present findings of ACA of papers of the latter group by means of an author co-citation map, displaying pivotal authors whose work was co-cited along with papers that indirectly cited Arf's papers. Our analysis also includes Arf's seminal paper on quadratic forms over a field of characteristic two (Arf invariant). We trace the direct influence of his paper by means of a pennant diagram based on ACA. Finally, we trace indirect influence of Arf's paper by calculating its single publication $b$ index based on its citing papers and discuss our findings.

\section{Citation Analysis}

Arf published a total of 23 papers between 1939 and 1966. ${ }^{[29]}$ The distribution of these papers by language is as follows: 12 in French, 6 in German, 4 in English, and 1 in Italian. Two of his English papers (\#1 and \#2) were listed in Thomson Reuters' citation indexes along with two English abstracts (\#3 and \#4), and two German papers (\#5 and \#6) whose titles were translated to English [Table 1]. Listed as \#5 in Table 1, Arf's paper (1941) on quadratic forms over a field of characteristic 2 is his most prominent work (cited 88 times) and will be analyzed further in this study. His papers were cited a total of 96 times, ${ }^{6}$

6. A cited reference search under "Arf $C^{*}$ " in Thomson Reuters' citation indexes produced a total of 153 citations to Arf's papers. Hisseminal work (Arf, 1941) was cited 105 times, not 88. Some citations were wrongly attributed to Arf's other papers. 
Table 1: Arf's papers and abstracts listed in Thomson Reuters' citation indexes

Arf C, Imre K, Ozizmir, E. On algebraic structure of cluster expansion in statistical mechanics. J Math Phys 1965;6:1179-and. (Times cited: 3)

Arf C. On methods of Rayleigh-Ritz-Weinstein. Proc Am Math Soc 1952;3:223-32. (Times cited: 1)

Arf C. On Rayleigh-Ritz-Weinstein method. Bull Am Math Soc 1951;57:269-70. (Times cited: 0)

Arf C. On a free boundary problem in elasticity. Bull Am Math Soc 1951;57:136. (Times cited: 0)

Arf C. Research on the quadratic form in the field of characteristics 2. (Teil I.). Journal für die Reine und Angewandte Mathematik 1941;183:148-67. (Times cited: 88)

Arf C. Research into pure deriven (sic) enhancements of discrete rated perfectcompunds (sic). Journal für die Reine und Angewandte Mathematik 1940;181:1-44. (Times cited: 4)

The query "Arf $C^{*}$ " was run on Thomson Reuters' databases SCI-EXPANDED, SSCI, A and HCI, CPCI-S, CPCI-SSH, BKCI-S, and BKCI-SSH using the all years (1898-2011) time span (on October 26, 2011)

thereby making Arf's $b$ index score 3 . This is by no means commensurate with his fame, however.

In spite of his many prominent papers with significant discoveries named after him, Arf's bibliometric profile is almost nonexistent. None of Arf's now-classic papers that appeared in non-English journals that influenced advanced mathematics profoundly was indexed in Thomson Reuters' citation indexes up until recent years. Uncitedness among mathematicians with Fields Medal (considered to be the Nobel Prize of mathematics) or even Nobel Laureates is not uncommon. ${ }^{[36]}$ Yet, this is not the case for Arf. Even though his works were not listed in citation indexes properly, a cited reference search under "Arf C*" produced a total of 153 citations. Arf's one paper ${ }^{[6]}$ alone received a total of 105 citations. $^{7}$ A paper written by Lipman on "Stable ideals and Arf rings" in 1971 was cited a total of 100 times. ${ }^{[10]}$ Lipman was referring to Arf's original paper that appeared in Proceedings of the London Mathematical Society in 1948, which is not listed in ISI indexes. ${ }^{[9]}$ Indirect citations to Arf's paper ${ }^{[0]}$ did not contribute to Arf's $h$ index score. This is mainly due to the fact that Arf's significant contributions were published before the ISI's citation indexes came into being and a great majority of them were not written in English language journals, and therefore, not indexed by ISI. One could only speculate as to how many citations Arf's original papers would have generated had they been properly listed in ISI indexes.

7. American Mathematical Society's Mathematical Reviews (MR) Citation Database on the Web (http://www.ams.org/mathscinet/) provides 38 citations to Arf's six papers including 26 citations to Arf. ${ }^{[6]}$ The MR database covers relatively current citations (year 2000 to present) (http:/ / www.ams.org/mathscinet/help/citation_ database_understanding.html).
Although Arf's papers are not properly listed in ISI's citation indexes and citations to them, therefore, did not count towards Arf's $h$ index score. Arf's influence can be observed further through paper titles that contain various references to Arf's works (e.g., "Arf invariant", "Arf rings", "Hasse-Arf theorem", and so on). We performed an advanced search in Thomson Reuters' citation indexes (1898-2011) $)^{8}$ and found a total of 45 papers (37 articles, 3 proceedings paper, 3 book chapters, 1 book, and 1 correction) with "Arf*" in their titles. ${ }^{9}$ Note that not all 45 papers contained references to Arf's works. In fact, only 15 of them did (a total of 16 citations). Arf's classic papers ${ }^{[6,9]}$ received eight and five citations, respectively. These 45 papers were mainly classified under Mathematics and were cited a total of 279 times ( $b$ index 9 , maximum citation per item: 97, average citation per item: 6.49).

We performed a topical search (TS) in Thomson Reuters' citation indexes and found an additional 52 papers with "Arf*" in their keywords (i.e., topics). ${ }^{10}$ Note that only nine out of 52 papers cited Arf's two papers. ${ }^{[6,9]}$ These 52 papers received a sum of 208 citations ( $b$ index 8 , maximum citation per item: 25 , average citation per item: 4.00).

Altogether, 97 papers with "Arf*" in their titles or topics (e.g., abstract keywords or keywords given by their authors) published between 1965 and 2011 were cited a total of 487 times ( $b$ index 11, average maximum citation per item: 97 , average citation per item: 5.13). It should be noted that 24 authors cited Arf's works 25 times, but the great majority of authors (69 out of 97) who referred to Arf's works in titles or keywords of their papers did not necessarily give due credit to Arf in their reference lists by properly citing Arf's papers. Apparently, they were either

8. We used the following query: $\mathrm{TI}=(\operatorname{arf}$ theorem $)$ OR TI $=(\operatorname{arf}$ invariant $\left.{ }^{*}\right)$ OR TI $=(\operatorname{arf}$ ring* $)$ OR TI $=(\operatorname{arf}$ propert $*)$ OR TI $=(\operatorname{arf}$ filtration*) OR TI $=($ arf semigroup* $)$ OR TI $=(\operatorname{arf}$ singularit* $)$ OR $\mathrm{TI}=($ arf equivalence $)$ OR TI $=($ arf closure* $)$. Irrelevant items were discarded.

9. The terms used in the titles of these papers are as follows: Arf invariant* (the most common one) or Arf's invariant, Hasse-Arf filtrations, Arf rings, Arf numerical semigroups, Arf semigroups, Arf functions, Arf characteristics of singularities, Arf closure, Arf equivalence, Arf-Kervaire invariant, Hasse-Arf theorem, and Hasse-Arf property.

10. Note that TS gets the keywords from titles, abstracts, and the author-assigned keywords. Therefore, we excluded the titles to find the unique items that would be retrieved only through keywords that come from abstracts or author-assigned keywords. In other words, these 52 papers do not overlap with the previous 43 papers that we identified through title search. 
unaware of the existence of Arf's papers or they did not cite them because Arf's papers became a part of "regular scientific discourse". ${ }^{[37]}$ Indeed, as Terzioğlu ${ }^{[38]}$ points out, Arf's name is so intertwined with mathematics, one needs to work hard to find citations to Arf's papers. Some authors using Arf invariant in their works seem to have referred to it as a mathematical symbol or notation without, perhaps, thinking that these three characters are actually the last name of a Turkish mathematician (Tosun Terzioğlu, personal communication, August 16, 2011). Papers on geometric, algebraic, or differential topology frequently refer to Arf invariant as $\operatorname{Arf}(X)$ or $\operatorname{Arf}(M)$, (here $X$ or $M$ stands for a manifold), Arf $(K)$ for a knot, and $\operatorname{Arf}(q)$ for a quadratic form. These various forms of use of Arf invariant in relevant papers are hardly reflected in papers' titles, keywords, or reference lists (Turgut Önder, personal communication, August 24, 2011). ${ }^{11}$

We know that ISI indexers tended to make "implicit" citations to works of art or musical scores within the arts and humanities papers "explicit" by indexing them accordingly. ${ }^{[3-41]}$ Such citations count towards one's cited references and possibly towards his/her $b$ index score. Yet, we are not aware of any Thomson Reuters's convention that makes implicit references in paper titles or topics explicit, thereby giving credit to those whose works get cited tacitly. An implicit reference made explicit by indexers within a paper is equal to one citation. But what about an implicit reference found in the title or topic of a paper? It would certainly be worth more than one citation. For instance, Sertö ${ }^{[42]}$ in his article on Arf rings cites no fewer than eight papers that were clearly based, at least in part, on Arf's work and the two of those papers have "Arf*" in their titles (excluding Lipman's oft-cited paper that we mentioned earlier).

Suppose that we decided to find out what would Arf's total influence be if we weighed the papers with "Arf*" in their titles (45) and keywords (52). Further, suppose that each paper with "Arf*" in its title and keywords list is worth 10 and 5 citations, respectively. This would give us a total of 710 citations to Arf's works. One would safely claim that Arf's $h$ index score would have been much higher if his works were listed in Thomson Reuters indexes and

11. We are most grateful to Professor Turgut Önder of the Middle East Technical University (Ankara, TR) who generously shared his knowledge of Arf invariant and references to it in various forms in the mathematics literature and provided pointers to the relevant monographs mentioned earlier in the literature review. citations to his works in paper titles and keywords were made explicit. ${ }^{12}$

\section{Author Co-citation Analysis}

We further analyzed the papers with "Arf*" in their titles and topics (i.e., keywords) using CiteSpace. We selected the most frequently cited $20 \%$ of those papers and used ACA to visualize them in 10 -year time slices starting from 1966 [Figure 2]. Clearly, Arf C is the centroid of such an author co-citation network with a large circle. CiteSpace has a feature for identifying pivotal nodes based on Freeman's betweenness centrality metric. ${ }^{[43,44]}$ "In a network, the betweenness centrality of a node measures the extent, to which the node plays a role in pulling the rest of nodes in the network together (p. 235-236)". ${ }^{[45] 13}$ Kaufman LH, Robertello RA, Milnor J, Serre JP, Kervaire MA, Browder W, Bass H, Adams JF, and Bourbaki N are the pivotal nodes in the network (depicted as large circles with dark rims). These pivotal nodes and many gray-colored ones not labeled represent very important mathematicians.

Dark-colored clusters of network indicate works that are published most recently (from 2006 to 2011). Relatively speaking, they make up a considerable part of the network. Gray-colored parts of network represent the time span from 1976 to 2005. In other words, Cahit Arf is still cited heavily in papers with "Arf*" in their titles and/or keywords, along with the great mathematicians of today.

As we indicated earlier, a cited reference search for "Arf $C^{*}$ " generated 153 results. His $b$ index based on citations to his works that are not listed in citation indexes would be 4 . Table 2 provides the names of ten famous mathematicians who were co-cited frequently with Arf along with their $b$ index scores.

Names in Table 2 come from the most frequently cited $20 \%$ of Arf's co-cited authors. ${ }^{14}$ For example, an award-winning mathematician Jean-Pierre Serre is on the top of the

12. For comparison, as of February 1, 2013, J Lipman wrote 35 papers (including the one on Arf rings receiving 100 citations) and were cited 679 times ( $b$ index 12).

13. Betweenness centrality measure is "the number of shortest paths that pass through a given node.... Betweenness gauges the extent, to which a node facilitates the flow in the network." ${ }^{[16]}$ (p. 443)

14. It should be noted that "Nicolas Bourbaki" (Bourbaki N) is actually a pseudonym referring to more than one author. For more information, see the Wikipedia article at http://en.wikipedia.org/ wiki/Nicolas_Bourbaki. 


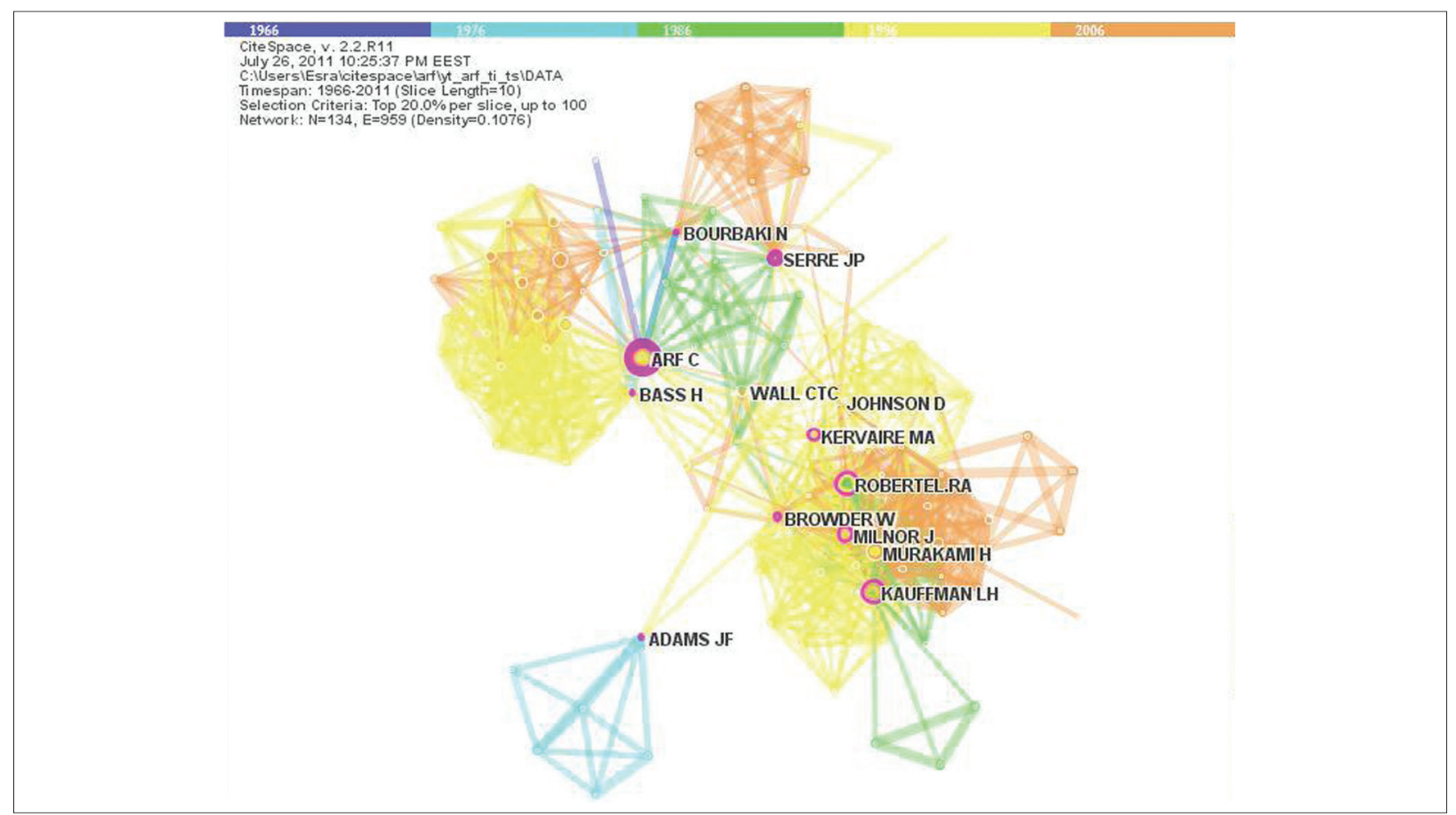

Figure 2: Author co-citation network (co-cited with authors of papers with "Arf*" in their titles/keywords)

Table 2: Co-cited author distributions

\begin{tabular}{lcc}
\hline Co-cited authors & Frequencies & $\boldsymbol{h}$ index scores \\
\hline Arf C & 146 & 1 \\
Serre JP & 30 & 23 \\
Witt E & 23 & (No ISI records under his name) \\
Bourbaki N & 21 & (No ISI records under his name) \\
Milnor J & 20 & 29 \\
Dieudonne J & 20 & 11 \\
Sah CH & 20 & 14 \\
Kervaire MA & 18 & 9 (with respect to 10 papers) \\
Omeara OT & 18 & 11 \\
Wall CTC & 18 & 25 \\
Kneser M & 14 & 8 \\
\hline
\end{tabular}

co-cited authors list with an $b$ index of 23 . He won the Abel Prize (2003), the Fields Medal (1954), the Wolf Prize in Mathematics (2000), and the Balzan Prize (1985). He also gave a speech about Cahit Arf in "Cahit Arf Seminars" in 2006 at the Middle East Technical University in Ankara, Turkey. J Milnor has an $b$ index of 29 and appeared in Arf's co-cited authors list, too. Witt and Kervaire are also on the above list. Witt, too, worked in Göttingen and is still being cited with his works on Witt algebra, Witt decomposition, Witt design (Witt geometry), Witt group, Witt index, Witt polynomial, Witt ring, Witt scheme, Witt's theorem, Witt vector, Bourbaki-Witt theorem, and Shirshov-Witt theorem. However, he has no record with his name in citation indexes, either. Due to Kervaire's later work, ${ }^{[46]}$ the Arf invariant is sometimes referred to as the Arf-Kervaire invariant because the Kervaire invariant itself is a quadratic form of the Arf invariant. Incidentally, the longstanding Arf-Kervaire problem about dimensions, in which there are framed manifolds of non-zero Kervaire invariant in algebraic topology, has been solved by three mathematicians in April 2009. ${ }^{[4] 15}$ Neither Arf nor Kervaire (who died in 2007) has lived long enough to see the solution of the Arf-Kervaire problem. A group of $20^{\text {th }}$ century mathematicians published nine important books under the pseudonym of Nicolas Bourbaki, presenting an exposition of modern advanced mathematics and receiving hundreds of citations to them. It should be pointed out that names that appeared in author co-citation network and top co-cited author distributions such as Serre, Milnor, Dieudonne, Kervaire, Kneser, Bass, Adams, and Witt are all well-known mathematicians.

We used co-citation statistics to draw a pennant diagram of Cahit Arf to trace his scientific influence further.

15. We thank Professor Turgut Önder for drawing our attention to the solution of the Arf-Kervaire invariant. Professor Önder points out that the solution of this almost 50 -year-old problem was one of the few outstanding developments in the mathematics world in 2009 (Turgut Önder, personal communication, August 24, 2011). For more on this, see Douglas Ravenel's web site at http://www. math.rochester.edu/u/faculty/doug/kervaire.html. 
What follows is a discussion of influence of Cahit Arf based on pennant diagram of his most significant work, "Untersuchungen über quadratische Formen in Körpern der Charakteristik 2. (Teil I).”[[]

The outcome of cited reference search under "Arf C*" (as of February 1, 2013) showed that his paper in which he first described the "Arf invariant" ${ }^{\text {[ }[]}$ was cited 105 times, constituting more than two-thirds of all citations to his works. We used $\operatorname{Arf}^{\left[{ }^{[6]}\right.}$ as the seed paper and identified the most highly cited $20 \%$ of references contained in those 103 papers citing Arf. ${ }^{[6]}$ We found that 234 papers were co-cited with Arf's seminal work (1941) at least once. To refine the resultant pennant diagram (otherwise it would be difficult to read the labels of nodes), we used 34 (out of 234) papers which were co-cited with Arf (1941) at least four times. Searching citation indexes, we found the $t f$ (items ranked) and $d f$ (items in file) values and used the following $t * i d f$ formula: ${ }^{[48]}$

weight $(i, j)=\left(1+\log \left(t f_{i, j}\right)\right) \log \left(N / d f_{i}\right)$ where, all term counts are greater than or equal to 1 , logarithms are based 10, and $N$ is the total number of items in the Thomson Reuters' data collection. ${ }^{[13] 16}$ Values for $x$ and $y$ axis in the pennant diagram in Figure 3 were calculated according to equation (1).(Appendix 1 gives the $t f$ and $i d f$ values sorted by $d f s$ and $t s)$.

Arf with his most significant paper (1941) appears at the tip of the pennant [Figure 3] at the right hand side as the seed term. ${ }^{[13]}$ (The years next to authors' names indicate publication years of relevant papers.) The seed term generates a bibliometric distribution, which predicts the relevance of any associated term with itself. Higher $f f$ and $i d f$ scores of any associated term will produce greater predicted relevance to the seed.

While the pennant diagram narrows through the right hand side, papers represented by points in Figure 3 become increasingly more relevant to that of Arf. ${ }^{[6]}$ Authors in

16. The number of items in ISI file is assumed to be 5 million.

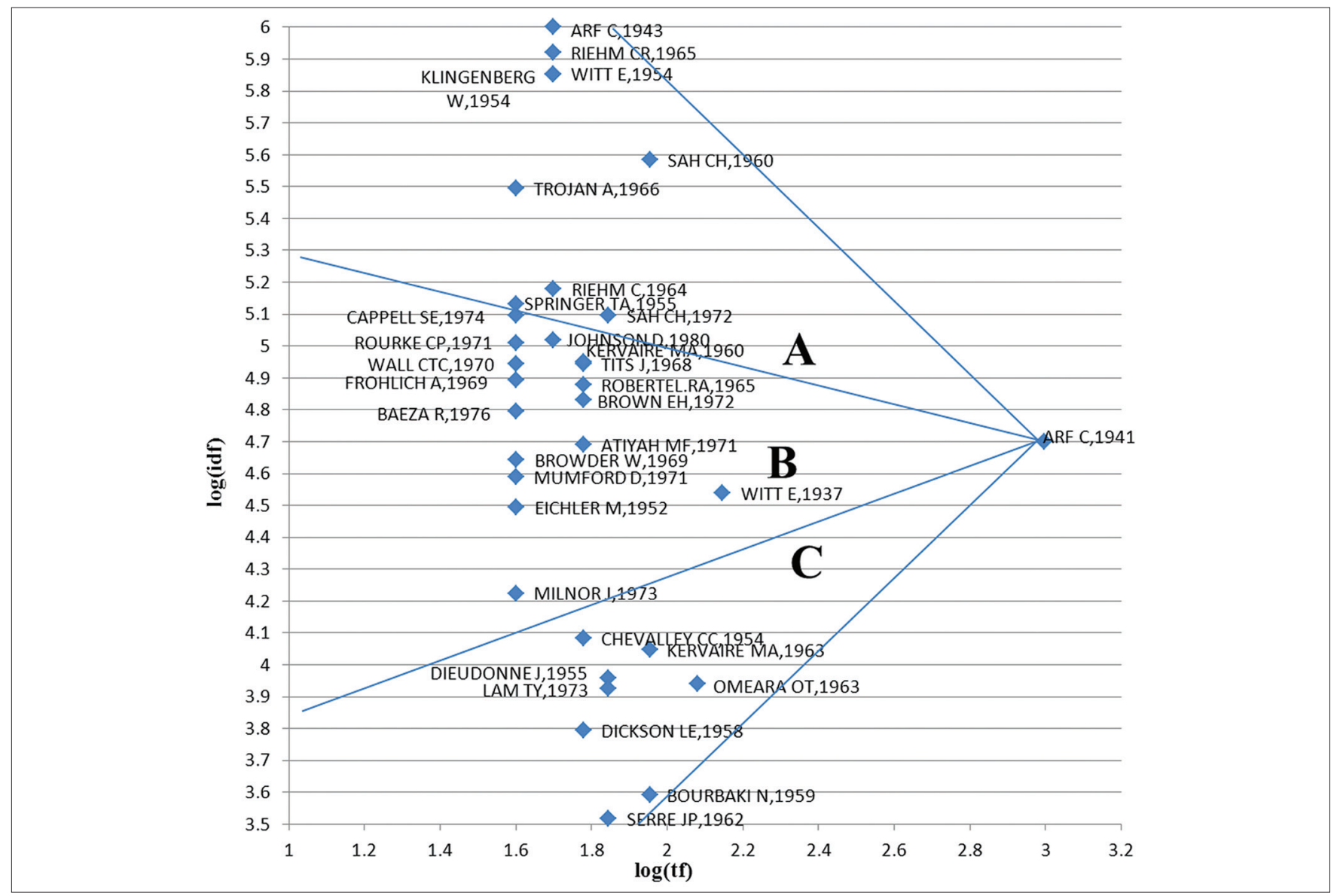

Figure 3: Pennant diagram of items co-cited with Arf's "Untersuchungen über quadratische Formen in Körpern der Charakteristik 2" (Arf, 1941). Only the first author's name is given in the pennant diagram for co-authored works 
the left-most column were co-cited at least four times with Arf's paper ${ }^{[6]}$ while the ones to its right were co-cited progressively more. For instance, Witt's 1937 paper was co-cited 12 times. (Figures in the $x$ and $y$ axis are both logged.) This is not a coincidence as Arf developed and completed Witt's work, as we explained earlier. Similarly, O'Meara's 1963 textbook Introduction to Quadratic Forms is the second highest co-cited work with $\operatorname{Arf}^{[6]}$ because it builds, at least in part, upon Arf's seminal work on the subject. ${ }^{17}$ Thus, it is relatively easier to discern the increasing relevance of papers as we move to the tip of the pennant.

White divided the pennant diagram into three (sections $\mathrm{A}$, $B$, and C) and interpreted the results from various different angles such as specificity of works (i.e., topicality), ease of processing, age and authority, fame, and so on. ${ }^{[13]}$ For instance, co-cited authors at the top (section A) of the pennant are topically more specific, and therefore, more relevant to that of $\operatorname{Arf}^{\left[{ }^{[6]}\right.}$ compared to the ones at the middle (section B) or bottom (section C) of the pennant. Similarly, co-cited authors in section A are juniors of Arf while the ones in Section B and $\mathrm{C}$ are his peers and seniors, respectively.

We interpret Arf's pennant diagram based on figures given in Table 3, which show how the idf portion of the formula affects ranking of term distribution. Since Thomson Reuters' cited reference strings combine both cited works and cited authors, we can use them to make judgments about papers, works, and their authors. ${ }^{[13]}$ Based on these judgments, we drew dividing lines between sections A, B, and C.

Note that all but one (Cappell SE) author referred to in Table 3 are placed in section A of Arf's pennant diagram

17. Note that references to authors and their works in the pennant diagram can be found in Appendix 1. depicted in Figure 3. They have higher $t f$ and $i d f$ values in that they are both topically relevant to that of $\operatorname{Arf}^{\left[{ }^{[6]}\right.}$ and their relevance can easily be discerned. Take, for instance, Arf's own work published in 1943 ("Untersuchungen über quadratische Formen in Körpern der Charakteristik 2. II. Über aritmetische Äquivalenz quadratischer Formen in Potenzreihenkörpern über einem vollkommenen Körper der Charakteristik 2"). It is shown at the top of the pennant diagram because it is a sequel of and complements Arf's original 1941 paper. Papers by Riehm CR, Klingenberg W, Witt E, and Sah CH at the top of section A is specifically about quadratic forms over a field of characteristic two, which is exactly the subject of Arf's paper. ${ }^{18}$ The topics of papers by remaining four authors (namely, Trojan A, Riehm C, Springer TA, and Sah C) in section A are all about quadratic forms (but not necessarily quadratic forms over a field of characteristic two) and, therefore, placed in the relatively lower parts of section A. ${ }^{19}$ Note that Chih-Han $\mathrm{Sah}$ (entered in two different forms in citation indexes as Sah CH 1960 and Sah C 1972 but corrected in the pennant diagram) is placed higher on top of the pennant diagram with his specific paper on quadratic forms over a field of characteristic two, whereas he is placed relatively lower with his more general paper on symmetric bilinear forms and quadratic forms. So, all nine papers (including Arf's

18. The titles of their papers are: "Integral representations of quadratic forms in characteristic 2" by Riehm CR (1965); "Über die Arfsche Invariante quadratischer Formen mod 2" by Klingenberg W (1954); "Über eine Invariante quadratischer Formen mod 2" by Witt E (1954); and "Quadratic forms over fields of characteristic-2" by Sah CH (1960).

19. The titles of their papers are: "Integral extension of isometries of quadratic forms over local fields" by Trojan A (1966); "On integral representations of quadratic forms over local fields" by Riehm C (1964); "Quadratic forms over fields with a discrete valuation," by Springer TA (1955), and "Symmetric bilinear forms and quadratic forms” by Sah C (1972).

Table 3: Ranks of the first ten co-cited items with Arf (1941) based on $t f$ and idf

\begin{tabular}{|c|c|c|c|c|c|c|c|}
\hline \multirow[t]{2}{*}{ Reference } & \multicolumn{3}{|c|}{ Frequency } & \multicolumn{4}{|c|}{ Sector \% } \\
\hline & $t f$ & $d f$ & $N$ & $(t f / d f) * 100$ & $t f^{\star} i d f$ & $\log (1+t f)$ & $\log (5 \mathrm{mil} / \mathrm{df})$ \\
\hline Arf C 1943 & 5 & 5 & $5 E+06$ & 28,316 & 10,194 & 1,699 & 6,000 \\
\hline Riehm CR 1965 & 5 & 6 & $5 E+06$ & 28,694 & 10,059 & 1,699 & 5,921 \\
\hline Klingenberg W 1954 & 5 & 7 & $5 \mathrm{E}+06$ & 29,023 & 9,946 & 1,699 & 5,854 \\
\hline Witt E 1954 & 5 & 7 & $5 E+06$ & 29,023 & 9,946 & 1,699 & 5,854 \\
\hline Sah CH 1960 & 9 & 13 & $5 E+06$ & 34,991 & 10,914 & 1,954 & 5,585 \\
\hline Trojan A 1966 & 4 & 16 & $5 E+06$ & 29,156 & 8,803 & 1,602 & 5,495 \\
\hline Riehm C 1964 & 5 & 33 & $5 E+06$ & 32,796 & 8,801 & 1,699 & 5,180 \\
\hline Springer Ta 1955 & 4 & 37 & $5 E+06$ & 31,225 & 8,220 & 1,602 & 5,131 \\
\hline Sah C 1972 & 7 & 40 & $5 E+06$ & 36,200 & 9,404 & 1,845 & 5,097 \\
\hline Cappell SE 1974 & 4 & 40 & $5 E+06$ & 31,432 & 8,166 & 1,602 & 5,097 \\
\hline
\end{tabular}

$t f=$ Term frequency, $d f=$ Document frequency; $i d f=$ Inverse document frequency 
sequel in 1943) in section A are highly relevant to Arf's original paper. ${ }^{[49]}$

One can easily see that authors in section $\mathrm{A}$ are juniors of Arf (not by age, perhaps, by the topic of their papers) in that they built on or they did further research on Arf invariant. Note that Witt whose 1937 paper was developed by $\mathrm{Arf}^{[6]}$ wrote a specific paper on Arf invariant later in 1954.

As we move down from section A to section $B$, the difference between the subject of Arf's paper and those in section B gets more difficult to discern because papers are no longer specifically on quadratic forms. For instance, Cappell's 1974 paper is not on quadratic forms ("Unitary Nilpotent Groups and Hermitian K-Theory 1"). We, therefore, drew the line between section A and B just above Cappell SE 1974 in Figure 3. In general, the relationship between Arf's original paper and those in sector B are not necessarily obvious. Yet, authors in section B can be considered peers of Arf in mathematics, and they were usually co-cited with Arf [Table 2]. We have already mentioned Witt E 1937 and Kervaire M 1960 earlier. Many of Arf's peers in section $\mathrm{B}$ are considered top mathematicians, some with high index scores, ${ }^{20}$ some (just like Arf himself) not properly represented in indexes (e.g., Kervaire M), ${ }^{21}$ or not represented at all (e.g., Witt E).

White ${ }^{[13]}$ considers cited authors in section $\mathrm{C}$ as "seniors, culture heroes (p. 556)," cited works as serials, generic titles, and world classics; and cited references as books and classic articles. The contributions of authors in section C of Arf's pennant diagram validate White's prediction in that they consist mostly of classic mathematics texts. Corps Locaux by Serre JP (1962), Algebre by Bourbaki N (1959), Linear Groups with an Exposition of the Galois Field Theory by Dickson LE (1958), Introduction to Quadratic Forms over Fields by Lam TY (1973), Introduction to Quadratic Forms by O'Meara (1963), La Géométrie Des Groupes Classiques by Dieudonne (1955), and Algebraic Theory SPI by Chevalley C (1954) are all placed at the bottom of Arf's pennant diagram along with a highly cited article (cited a total of 449 times as of July 25, 2011) by Kervaire MA (and Milnor J) entitled "Groups of Homotopy Spheres: I". These authors can be considered seniors of Arf as they authored highly regarded textbooks in mathematics.

20. For example, Atiyah MF with $b$ index 34, Milnor J with 29, Wall CTC with 25, Cappell SE with 17, and Frohlich A with 16.

21. Kervaire $M$ has an $b$ index score of 9 with 10 of his papers being listed in citation indexes.

\section{Single Publication $h$ Index}

After the assessment of direct influence of Arf's seed work on Arf invariants ${ }^{[6]}$ based on ACA, we wanted to ascertain indirect influence of same work based on its citing papers using Schubert's single publication $b$ index (defined as "the set of papers citing the work in question"). ${ }^{[15]}$ We used Google Scholar database and Thor and Bornmann's ${ }^{[26]}$ readily available web application to calculate the $h$ index of Arf's paper. The application retrieved a total of 95 papers from Google Scholar citing Arf. ${ }^{[6]}$ Figure 4 provides the partial list of citing papers with their bibliographic information (author, title, and the publication year) and the number of times they were cited. Citing papers were cited between 1009 and 0 times. Thirty-nine out of 95 citing papers $(41.05 \%)$ were never cited at all. The rest (56) were cited a total of 3,346 times. The most current paper citing Arf ${ }^{[6]}$ is dated $2011 .{ }^{22}$ It is interesting to note that all but one citing papers were written in German, indicating the international coverage of Google Scholar database.

It should be noted that not all papers citing $\operatorname{Arf}^{[6]}$ are correctly identified by Google Scholar. Eleven papers in the list citing $\mathrm{Arf}^{\left[{ }^{[6]}\right.}$ were published before 1941. An additional 11 papers lacked publication year information. These 22 papers were cited a total of 811 times (more than one-fifth of all indirect citations). We have not checked the papers with publication year information furnished to see if they are correctly identified by Google Scholar matching algorithms. ${ }^{[50,51] 23}$

Notwithstanding the limitations of Google Scholar, single publication $b$ index of Arf's paper ${ }^{[6]}$ was calculated as 29 by Thor and Bornmann's ${ }^{[26]}$ web application. Figure 5 provides citation distribution graph of 95 papers citing Arf ${ }^{[[]]}$excluding self-citations.

The graph plots the citing papers sorted by the number of times they were each cited in the $x$ axis and number of citations in $y$ axis. Note that the area plotted is divided into three sections ( $h^{2}$ upper, $b^{2}$ center, and $h^{2}$ lower) proposed by Bornmann et al..$^{[52]}$ These metrics and what

22. Professor Turgut Önder points out that the solution of the Arf-Kervaire invariant has brought the Arf invariant into the fore once again and will likely increase citations to Arf's original 1941 paper as well as to current papers discussing the solution, thereby increasing Arf's original paper's single publication $b$ index (Turgut Önder, personal communication, August 24, 2011).

23. The shortcomings of Google Scholar's matching algorithms are well documented in the literature. See, for example, Jacsó ${ }^{[50]}$ and Bar-Ilan. ${ }^{[51]}$ 
Tonta and Özkan Çelik: Cahit Arf and his influence in mathematics

\begin{tabular}{|c|c|c|c|c|c|}
\hline \multicolumn{3}{|c|}{ Untersuchungen über quadratische Formen in Körpern der Charakteristik 2} & \multicolumn{3}{|c|}{ Search } \\
\hline \multicolumn{6}{|c|}{ Search result for Untersuchungen über quadratische Formen in Körpern der Charakteristik 2} \\
\hline$\square$ & title & authors & year & citatio... & \\
\hline 回 & Theorie der konvexen Körper & W Fenchel & 1948 & 1009 & a \\
\hline$\square$ & Vorlesungen über Geometrie der Algebren & W Benz & 1973 & 302 & $=$ \\
\hline$\square$ & Algebraische Konstruktion reeller Körper & E Artin, O Schreier & 1927 & 201 & \\
\hline$\square$ & Quadratische Formen in beliebigen Körpern & A Pfister & 1966 & 183 & \\
\hline$\square$ & Zur Theorie der Absorptionsspektren fester Körper & R Peierls & 1932 & 166 & \\
\hline$\square$ & Untersuchungen über quadratische Formen in Körpern der Charakteristik 2 & CArf & 1941 & 161 & \\
\hline$\square$ & Diophantische approximationen: Eine einführung in die zahlentheorie & H Minkowski & 1907 & 140 & \\
\hline$\square$ & Angeordnete Strukturen & S Priess-Crampe & 1983 & 140 & \\
\hline$\square$ & Einführung in die reelle Algebra & II Knebusch, C Scheiderer & 1989 & 120 & \\
\hline$\square$ & Gesammelte Abhandlungen & FG Frobenius & 1968 & 103 & \\
\hline$\square$ & Einführung in die endliche Geometrie: II. Projektive Räume & A Beutelspacher & 1983 & 94 & \\
\hline$\square$ & Analytische Arithmetik der positiven quadratischen Formen & E Hecke & 1940 & 94 & \\
\hline$\square$ & Quadratische Formen über $Q$ & M Kneser, R Scharlau & 2002 & 89 & \\
\hline 回 & Eine Kennzeichnung der reell abgeschlossenen Körper & E Artin, O Schreier & 1927 & 80 & \\
\hline$\square$ & Specialization of quadratic and symmetric bilinear forms and a norm theorem & In Knebusch & 1973 & 52 & 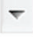 \\
\hline 4 & III & & & 1 & \\
\hline
\end{tabular}

Figure 4: Search results for Arf's seminal paper, “Untersuchungen über quadratische Formen in Körpern der Charakteristik 2" based on Google Scholar data (February 1, 2013)

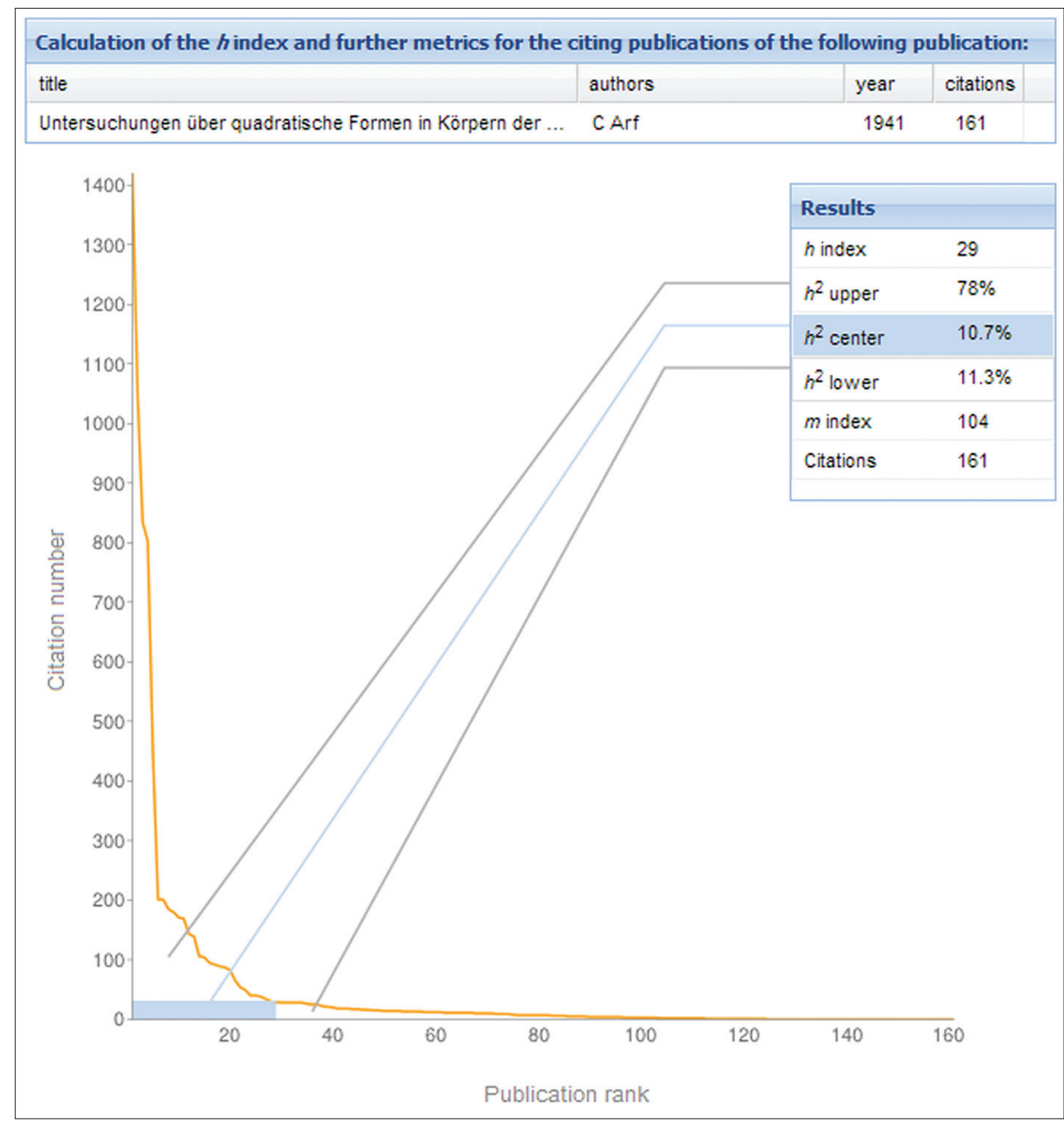

Figure 5: Single publication $b$ index for Arf's seminal paper, "Untersuchungen über quadratische Formen in Körpern der Charakteristik 2. (Teil 1)" (February 1, 2013) 
they represent are delineated in the single publication $b$ index web application site (http://labs.dbs.uni-leipzig. $\mathrm{de} / \mathrm{gsh} /$ ) as follows:

They allow quantification of three areas within a distribution of citing publications of one single publication: The low impact area ( $h^{2}$ lower), the area captured by the $b$ index ( $b^{2}$ center), and the area of publications with the highest visibility ( $b^{2}$ upper). The citing publications of one single publication (with the same $b$ index) may be dominated by low-impact citing publications (reflected by a high percentage for $b^{2}$ lower) or by high-impact citing publications (reflected by a high percentage for $b^{2}$ upper). The $\mathrm{m}$ index is the median number of citations received by citing publications in the Hirsch core; this is the citing publications ranking smaller than or equal to $h \cdot{ }^{[53]}$

We then calculated the single publication $b$ index for the first 50 papers with the highest number of citations (ranging between 1,318 and 65). We located only 15 papers whose single publication $b$ index scores were higher than that of Arf's 1941 paper. ${ }^{[6124}$ The number of citations measures the direct influence of his paper while single publication $b$ index measures its indirect influence.

This small scale experiment shows that Arf's paper has been among the top 15 papers in terms of both total number of citations it generated and its single publication $b$ index score. This is a remarkable achievement for a scientist, especially when one considers the fact that Arf's paper has never been listed in Thomson Reuters' citation indexes.

\section{CONCLUSIONS AND FURTHER RESEARCH}

Cahit Arf's contributions were not properly listed in citation indexes, and his $b$ index score or any other bibliometric indicator cannot, therefore, be calculated properly. In this study, we used SNA and author co-citation network of Arf to study his overall scientific influence retrospectively. Using White's approach, we drew Arf's pennant diagram on the basis of his author co-citation map to reveal his scientific impact (despite the foggy retrospective data that could be gathered from Thomson Reuters). We also calculated the single publication $b$ index score for his most significant paper. $^{[6]}$

Arf's cited references and paper titles or keywords with Arf's contributions to mathematics (e.g., "Arf invariant"

24. Note that rest of the papers are highly unlikely to produce single publication $b$ index scores over 29 . or "Arf rings") indicate that Arf is still being cited heavily, despite the fact that his last contribution was in 1960s. This is further confirmed by results of SNA (1966-2011) as Arf continues to play a prominent role in mathematics. Moreover, the pennant diagram and single publication $h$ index score based on Arf's seminal paper ${ }^{[6]}$ clearly shows his overarching influence on generations of mathematicians. His paper on Arf invariants has been among the top 15 papers having both direct and indirect influence in mathematics and related fields such as knot theory.

Findings obtained through SNA and the pennant diagram seemed to be similar in that, some pivotal authors on the co-citation network appeared as peers of Arf in the pennant diagram. However, further work is needed to compare results of two methods more comprehensively. As Arf's pennant diagram is based on a single article, we may be missing some crucial authors or works. Further work is also needed to find out if Arf's implicit citations (from titles and keywords of papers) can be incorporated in such analyses using a somewhat different approach. This may provide a fuller picture of Arf's scientific influence.

This study clearly shows that White's ${ }^{[13]}$ approach and pennant diagrams can be used to study the impact of authors who are no longer active or when their $b$ index scores cannot be calculated on the basis of available data. Once a proper method is developed in the future to incorporate implicit citations to pennant diagrams as co-citations, they can be used to calculate authors' retrospective $b$ index scores. One can conjecture that explicit citations in reference lists of papers would be placed in section A of a pennant diagram, while implicit citations contained in paper titles and keywords be placed in section B and C, respectively. Further research is needed to validate this conjecture.

Similarly, Schubert's single publication $h$ index ${ }^{[15]}$ can be used to study indirect influence of individual papers. Yet, we need cleaner data and better matching algorithms to attribute the citations correctly to the seed paper and to improve the accuracy of scores of single publication $b$ index. Simple checks like the comparison of publication years will certainly be of help in this respect. Furthermore, the calculation of indirect influence of a paper can further be refined by assigning weights to indirect citations, as suggested by Rousseau ${ }^{[54]}$ long before the $h$ index existed. 


\section{ACKNOWLEDGEMENTS}

We are grateful to Professors Ronald Rousseau of KHBO (Ostend, BE), Tosun Terzioğlu of Sabanc1 University (İstanbul, TR), Turgut Önder of the Middle East Technical University (Ankara, TR), Dr. Umut Al, Güleda Düzyol and Zümra Kavafoğlu of Hacettepe University (Ankara, TR) for carefully reading the earlier drafts of this paper and providing generous comments. We also thank Merve Okur of Mikro Bilgi Kayıt ve Dağıtım A.Ş. (Ankara, TR) for research assistance.

\section{REFERENCES}

1. Terzioğlu T, Yılmaz A. Anlamak Tutkunu Bir Matematikçi: Cahit Arf. Ankara: TÜBA; 2005.

2. Reisman A. (2007, June 12). Turkey's invitations to Nazi persecuted intellectuals circa 1933: A bibliographic essay on history's blind spot. Available from: http://ssrn.com/abstract=993310 [Last accessed on July 4, 2013].

3. Reisman A. Turkey's Modernization: Refugees from Nazism and Atatürk's Vision. Washington, DC: New Academia Publishers; 2006.

4. O'Connor JJ, Robertson EF. (1998). Arfbiography, MacTutor History of Mathematics archive. Available from: http://www-history.mcs. st-andrews.ac.uk/Biographies/Arf.html [Last accessed on July 4, 2013].

5. Witt E. Theorie der quadratischen Formen in beliebigen Körpern. Journal für die Reine und Angewandte Mathematik 1937; 176:31-44.

6. Arf C. Untersuchungen über quadratische Formen in Körpern der Charakteristik 2. (Teil I.) Journal für die Reine und Angewandte Mathematik 1941;183:148-67.

7. Ikeda MG. Cahit Arf's contribution to algebraic number theory and related fields. Turk J Math 1998;22:1-14.

8. Önder T. Arf invariant and its applications in topology. In: Arf C, editor. The Collected Papers of Cahit Arf. İstanbul: Türk Matematik Derneği; 1990. p. 413-5.

9. Arf C. Une interpretation algébrique de la suite des ordres de multiplicité d'une branche algébrique. Proceedings of the London Mathematical Society, Series 2 1948;50:256-87.

10. Lipman J. Stable ideals and Arf rings. Am J Math 1971;93:649-85.

11. Sertöz S. Arf rings and characters. Note di Matematica 1997;14:251-61.

12. O'Connor JJ, Robertson EF. Arf biography, MacTutor History of Mathematics archive. 1998. [Last accessed on July 4th, 2013, from http://www-history.mcs.st-andrews.ac.uk/Biographies/Arf.html]

13. White HD. Combining bibliometrics, information retrieval, and relevance theory. Part 1: First examples of a synthesis. J Am Soc Inf Sci 2007a;58:536-59.

14. White HD. Pennants for Strindberg and Persson. In: Celebrating Scholarly Communication Studies: A Festschrift for Olle Persson at his $60^{\text {th }}$ Birthday. Special Volume of the E-Newsletter of the International Society for Scientometrics and Informetrics 2009;5-S: 71-83.

15. Schubert A. Using the $h$-index for assessing single publications. Scientometr 2009;78:559-65.

16. Otte E, Rousseau R. Social network analysis: A powerful strategy, also for the information sciences. J Inf Sci 2002;28:443-55.

17. Chen C. CiteSpace II: Detecting and visualizing emerging trends and transient patterns in scientific literature. J Am Soc Inf Sci 2006;57:359-77.
18. White HD, Griffith BC. Author co-citation: A literature measure of intellectual structure. J Am Soc Inf Sci 1981;32:163-71.

19. McCain KW. Longitudinal author cocitation mapping: The changing structure of macroeconomics. J Am Soc Inf Sci 1984;35:351-9.

20. McCain KW. Mapping authors in intellectual space. A technical overview. J Am Soc Inf Sci 1990;41:433-43.

21. White HD, McCain KW. Visualizing a discipline: An author co-citationanalysis of information science, 1972-1995. J Am Soc Inf Sci 1998;59:2146-55.

22. White HD. Combining bibliometrics, information retrieval, and relevance theory. Part 2: Some implications for information science. J Am Soc Inf Sci 2007b;58:583-605.

23. White HD. Some new tests of relevance theory in information science. Scientometr 2010;83:653-67.

24. Sparck Jones SK. A statistical interpretation of term specificity and its application to retrieval. J Doc 1972;28:11-21.

25. Hirsch JE. An index to quantify an individual's scientific research output. Proceedings of the National Academy of Sciences of the United States of America 2005;102:16569-72.

26. Thor A, Bornmann L. The calculation of the single publication $h$ index and related performance measures: A web application based on Google Scholar data. Online Inf Rev 2011;35:291-300.

27. Sertöz AS. A scientific biography of Cahit Arf (1910-1997). (Unpublished manuscript); 2011.

28. Bilim ve Teknik, 1998 February. Special issue on Cahit Arf. No. 363. Available from: http://www.biltek.tubitak.gov.tr/bdergi/ozel/arf/ default.html [Last accessed on July 4th, 2013].

29. Arf C. The Collected Papers of Cahit Arf. İstanbul: Türk Matematik Derneği; 1990.

30. Snaith VP. Stable Homotopy Around the Arf-Kervaire Invariant. Basel: Birkhäuser; 2009.

31. Klaus S. Brown-Kervaire Invariants. PhD Thesis. Shaker, Aachen: Universität Mainz; 1995.

32. Scorpan A. The Wild World of 4-manifolds. Providence, RI: American Mathematical Society; 2005.

33. Kirby RC. The Topology of 4-manifolds. Berlin: Springer-Verlag; 1989.

34. Browder W. editor. Algebraic Topology and Algebraic K-theory: Proceedings of a Symposium in Honor of John C. Moore. Princeton, NJ: Princeton University Press; 1987.

35. Lorenz F, Roquette P. On the Arf invariant in historical perspective. Mathematische Semesterberichte 2010;57:73-102.

36. Egghe L, Guns R, Rousseau R. Thoughts on uncitedness: Nobel laureates and fields medalists as case studies. J Am Soc Inf Sci 2011;62:1637-44.

37. Tonta $Y$, Darvish HR. Diffusion of latent semantic analysis as a research tool: A social network analysis approach. J Informetr 2010;4:166-74.

38. Terzioğlu T. (1998 February). Sunuş yerine (Introduction to the special issue on Cahit Arf). Bilim ve Teknik No. 363. Available from: http://www.biltek.tubitak.gov.tr/bdergi/ozel/arf/sunus.html [Last accessed on July 4th, 2013].

39. AI U, Şahiner M, Tonta Y. Arts and humanities literature: Bibliometric characteristics of contributions by Turkish authors. J Am Soc Inf Sci 2006;57:1011-22.

40. Garfield E. Is information retrieval in the arts and humanities inherently different from that in science? The effect that ISI's citation index for the arts and humanities is expected to have on future scholarship. Libr Q 1980;50:40-57.

41. Stern M. Characteristics of the literature of literary scholarship. Coll Res Libr 1983;44:199-209.

42. Sertöz S. On Arf rings. In: Arf C, editor. The Collected Papers of Cahit Arf. 1990. p. 416-9.

43. Freeman LC. A set of measures of centrality based on betweenness. Sociometry 1977;40:35-41.

44. Chen $\mathrm{C}$. The centrality of pivotal points in the evolution of scientific 
networks. In: Proceedings of the $10^{\text {th }}$ International Conference on Intelligent User Interfaces (IUI 2005), January 09-12, 2005, San Diego, California, USA (pp. 98-105). ACM Press. Available from: http://citeseer.ist.psu.edu/viewdoc/summary? [Last accessed on July 4th, 2013].

45. Chen C, Song IY, Yuan X, Zhang J. The thematic and citation landscape of Data and Knowledge Engineering (1985-2007). Data Knowl Eng 2008;67:234-59.

46. Kervaire M. A manifold which does not admit any differentiable structure. Commentarii Mathematici Helvetici 1960;34: 257-70.

47. Hill MA, Hopkins MJ, Ravenel DC. (2010) On the non-existence of elements of Kervaire invariant one. Available from: http://arxiv. org/PS_cache/arxiv/pdf/0908/0908.3724v2.pdf [Last accessed on July 4th, 2013].

48. Manning $C D$, Schütze $H$. Foundations of Statistical Natural Language Processing. Cambridge, MA: MIT Press; 2000.

49. Hsia JS. A note on the integral equivalence of vectors in characteristic 2. Mathematische Annalen 1968;179:63-9.
50. Jacsó P. The pros and cons of computing the h-index using Google Scholar. Online Inf Rev 2008;32:437-52.

51. Bar-llan J. Which h-index? A comparison of WoS, Scopus and Google Scholar. Scientometrics 2008;74:257-71.

52. Bornmann L, Mutz R, Daniel HD. The $h$ index research output measurement: Two approaches to enhance its accuracy. J Informetr 2010;4:407-14.

53. Bornmann L, Mutz R, Daniel HD. Are there better indices for evaluation purposes than the $h$ index? A comparison of nine different variants of the $h$ index using data from biomedicine. JAm Soc Inf Sci 2008;59:830-7.

54. Rousseau R. The Gozinto theorem: Using citations to determine influences on a scientific publication. Scientometr 1987;11:217-29.

How to cite this article: Tonta Y, Özkan Çelik AE. Cahit Arf: Exploring his scientific influence using social network analysis, author co-citation maps and single publication $\mathrm{h}$ index1. J Sci Res 2013;2:37-51.

Source of Support: Nil, Conflict of Interest: None declared

Appendix 1: tf and idf values for authors in Arf's pennant diagram

\begin{tabular}{|c|c|c|c|}
\hline & $\begin{array}{c}\text { Weight } \\
\text { tf*idf }\end{array}$ & $\begin{array}{c}\log (t f) \\
\log (1+t f)\end{array}$ & $\begin{array}{c}\log (i d f) \\
\log (5 \mathrm{mil} / \mathrm{df})\end{array}$ \\
\hline Arf C 1941, J Reine Angew Math, V 183, P 148 & 14.076 & 2.996 & 4.699 \\
\hline \multicolumn{4}{|l|}{ Sector A } \\
\hline Arf C 1943, Rfsui A, V 8, P 297 & 10.194 & 1.699 & 6.000 \\
\hline Riehm Cr 1965, Am J Math, V 87, P 32 & 10.059 & 1.699 & 5.921 \\
\hline Klingenberg W 1954, J Reine Angew Math, V 193, P 121 & 9.946 & 1.699 & 5.854 \\
\hline Witt E 1954, J Reine Angew Math, V 193, P 119 & 9.946 & 1.699 & 5.854 \\
\hline Sah Ch 1960, Am J Math, V 82, P 812 & 10.914 & 1.954 & 5.585 \\
\hline Trojan A 1966, Canadian J Math, V 18, P 920 & 8.803 & 1.602 & 5.495 \\
\hline Riehm C 1964, Am J Math, V 86, P 25 & 8.801 & 1.699 & 5.180 \\
\hline Springer Ta 1955, Indag Math, V 17, P 352 & 8.220 & 1.602 & 5.131 \\
\hline Sah C 1972, J Algebra, V 20, P 144 & 9.404 & 1.845 & 5.097 \\
\hline \multicolumn{4}{|l|}{ Sector B } \\
\hline Cappell Se 1974, B Am Math Soc, V 80, P 1117 & 8.166 & 1.602 & 5.097 \\
\hline Johnson D 1980, J Lond Math Soc, V 22, P 365 & 8.525 & 1.699 & 5.018 \\
\hline Rourke CP 1971, Ann Math, V 94, P 397 & 8.024 & 1.602 & 5.009 \\
\hline Kervaire M 1960, Comment Math Helv, V 34, P 257 & 8.803 & 1.778 & 4.951 \\
\hline Tits J 1968, Invent Math, V 5, P 19 & 8.790 & 1.778 & 4.943 \\
\hline Wall CTC 1970, Proc Camb Philos S-M, V 67, P 243 & 7.919 & 1.602 & 4.943 \\
\hline Frohlich A 1969, J Algebra, V 12, P 79 & 7.839 & 1.602 & 4.893 \\
\hline Robertel RA 1965, Commun Pur Appl Math, V 18, P 543 & 8.676 & 1.778 & 4.879 \\
\hline Brown EH 1972, Ann Math, V 95, P 368 & 8.588 & 1.778 & 4.830 \\
\hline Baeza R 1976, Lect Notes Math, V 655, P & 7.683 & 1.602 & 4.796 \\
\hline Atiyah MF 1971, Ann Sci Ecole Norm S, V 4, P 47 & 8.340 & 1.778 & 4.690 \\
\hline Browder W 1969, Ann Math, V 90, P 157 & 7.437 & 1.602 & 4.642 \\
\hline Mumford D 1971, Ann Sci Ecole Norm S, V 4, P 181 & 7.351 & 1.602 & 4.588 \\
\hline Witt E 1937, J Reine Angew Math, V 176, P 31 & 9.738 & 2.146 & 4.538 \\
\hline Eichler M 1952, Quadratische Formen, V, P & 7.201 & 1.602 & 4.495 \\
\hline Milnor J 1973, Symmetric Bilinear F, V, P & 6.768 & 1.602 & 4.225 \\
\hline \multicolumn{4}{|l|}{ Sector C } \\
\hline Chevalley CC, 1954, Algebraic Theory Spi, V, P & 7.260 & 1.778 & 4.083 \\
\hline Kervaire Ma 1963, Ann Math, V 77, P 504 & 7.908 & 1.954 & 4.047 \\
\hline Dieudonne J 1955, Geometrie Groupes CI, V, P & 7.303 & 1.845 & 3.958 \\
\hline Omeara Ot 1963, Intro Quadratic Form, V, P & 8.191 & 2.079 & 3.939 \\
\hline Lam Ty 1973, Algebraic Theory Qua, V, P & 7.244 & 1.845 & 3.926 \\
\hline
\end{tabular}




\section{Appendix 1: Contd/-}

\begin{tabular}{lccc} 
& $\begin{array}{c}\text { Weight } \\
\text { tf*idf }\end{array}$ & $\begin{array}{c}\log (\text { tf }) \\
\log (\mathbf{1 + t f})\end{array}$ & $\begin{array}{c}\text { (idf) } \\
\text { log (5 mil/df) }\end{array}$ \\
\hline Dickson Le 1958, Linear Groups Exposi, V, P & 6.749 & 1.778 & 3.795 \\
Bourbaki N, 1959, Algebre, Ch 9, P & 7.021 & 1.954 & 3.593 \\
Serre JP 1962, Corps Locaux, V, P & 6.492 & 1.845 & 3.518 \\
\hline
\end{tabular}

For papers having more than one authors, only the first author's name is provided above in the first column 